\title{
Emg Signal Classification Using Fuzzy Logic
}

\author{
O. Ülkir, G. Gökmen and E. Kaplanoğlu
}

\begin{abstract}
Electromyography (EMG) signals are an important technique in the control applications of prostatic hand. These signals, which are measured from the skin surface, are used to perform movements such as wrist flexion / extension, forearm supination / pronation and hand opening / closing of prosthetic devices. In this study, root mean square, waveform length and kurtosis methods were applied to extracted EMG signals from flexor carpi radialis and extensor carpi radialis muscles by using two channel surface electrodes. A fuzzy logic based classification method has been applied to classify the extracted signal features. With this method, classification for different gripping movements has been successfully accomplished.
\end{abstract}

Index Terms-Surface EMG, fuzzy logic, feature extraction, EMG classification.

\section{INTRODUCTION}

$\mathrm{E}$ LECTROMYOGRAM (EMG) is defined as muscle contraction resulting because of bioelectric signals. The source of those bioelectric signals is various electrochemical processes occurring in the body. Data logging of electrical signals in the muscles, provides important information in the diagnosis of abnormalities in both motor system and muscles [1].

EMG signals can be obtained by placing surface electrodes on the muscles or needle electrodes into the muscle tissue. Although differences between the needle and surface electrodes are not of a significant degree, the surface electrodes are preferred over the needle electrode. The most important reason for this is to be a safe and non-invasive measurement method [2].

EMG signals are used in medicine for the diagnosis of muscle and nerve disorders, besides that they are also used in engineering as an input for modelling of mechanical systems such as artificial limbs and also in prosthesis control engineering are used [3].

O. ÜLKIR, Department of Mechatronics Engineering, Technology Faculty, University of Marmara, Istanbul, Turkey, (e-mail: osman.ulkir@marmara.edu.tr). (iD

G. GÖKMEN, Department of Mechatronics Engineering, Technology Faculty, University of Marmara, Istanbul, Turkey, (e-mail: gokhang@ marmara.edu.tr).

E. KAPLANOĞLU, Department of Mechatronics Engineering, Technology Faculty, University of Marmara, Istanbul, Turkey, (e-mail: ekaplanoglu@marmara.edu.tr).

Manuscript received May 16, 2017; accepted August 11, 2017. DOI: $10.17694 /$ bajece.337941
Because the first EMG controlled prosthetics had only one grasping ability, they were only able to do open-close functions or simple proportional control techniques [4]. The multi-grasp prosthetics used today require more complex control methods, and therefore, the need for classification of EMG signals used for control arises.

An EMG signal from a muscle which performs different functions has only one pattern for a specific function. This pattern contains information about the direction of motion and the action's speed. To successfully control the prosthetic, this patterns must be classified correctly. A pattern recognition system is made up of a few steps: data acquisition, feature extraction selection, determination of the classification algorithm, and designing the classifier [5].

For classification it is necessary to determine what type of EMG signal from the muscles is recorded as the result of the movement. For this, the EMG signal must be classified after filtering and the appropriate signal must be matched to the appropriate movement. There are many methods for classifier design such as heuristic approach, explicit approach, statistical approach, artificial neural networks, support vector machines and fuzzy approach. [6-9]. Fuzzy logic method is a more preferred technique for classifier because biological markers don't repeat and sometimes show features beyond expectations [6]. In the literature, fuzzy logic classification method is frequently used in prosthetic hand applications [9-11]. In such studies, it is aimed to improve the results by applying different self-extracting methods. In this study, three different qualitative inference methods were applied at the same time in order to successfully perform the classification process.

The aim of this study is to distinguish different gripping movements for prosthetic hand applications. To distinguish these movements, it is necessary to classify signals received with surface EMG electrodes. In this study, fuzzy logic classification method is used. In order for the fuzzy logic classification method to be successful, the attributes used as input signals must be well chosen.

\section{METHODOLOGY}

The analysis of EMG signal consist of several steps, such as data acquisition, pre-processing, feature extraction and classification algorithm [12]. Figure 1 shows the block diagram of classification method. The EMG signals, received from the data collection card from the two different muscle groups, were passed through the preprocessing process for their feature extraction. In this study, three feature extraction methods were used. Then the fuzzy logic based classification method was applied. 
Be aware of the different meanings of the homophones "affect" (usually a verb) and "effect" (usually a noun), "complement" and "compliment," "discreet" and "discrete," "principal” (e.g., "principal investigator") and "principle" (e.g., "principle of measurement"). Do not confuse "imply" and "infer."

Prefixes such as "non," "sub," "micro," "multi," and "ultra" are not independent words; they should be joined to the words they modify, usually without a hyphen [2-4]. There is no period after the "et" in the Latin abbreviation "et al." (it is also italicized). The abbreviation "i.e.," means "that is," and the abbreviation "e.g.," means "for example" (these abbreviations are not italicized).

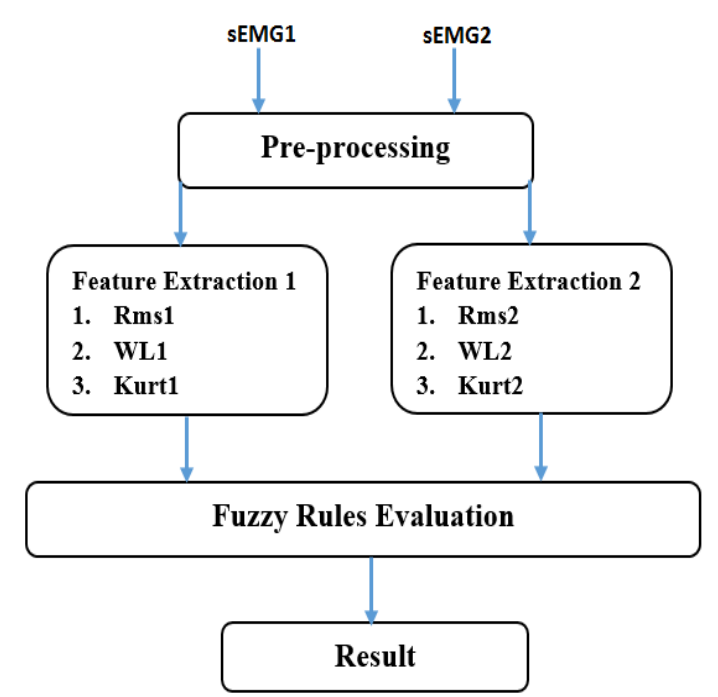

Fig.1. The block diagram of fuzzy logic classification method

\section{A. sEMG Data Acquisition}

EMG signals were obtained using surface electrodes by Bitalino EMG sensor. Four Ag-AgCI surface electrodes and one reference electrode is used, including two for each muscle. In this study, we focus on the wrist muscles which are the flexor carpi radialis (FCR) and extensor carpi radialis (ECR). These signals were given in Figure 3. Figure 2 shows the surface electrodes placed on the muscle surface.

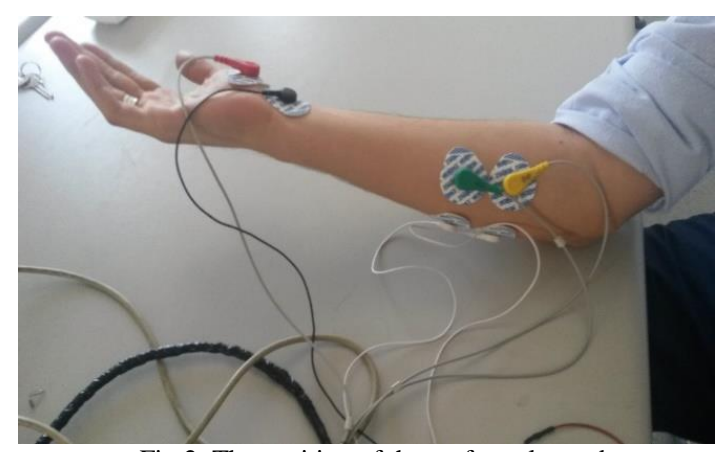

Fig.2. The position of the surface electrodes

The sampling frequency was set at $2 \mathrm{kHz}$ using Arduino Uno programming card. EMG signals were recorded for 10 seconds, 5 seconds of flexion and 5 seconds of extension.
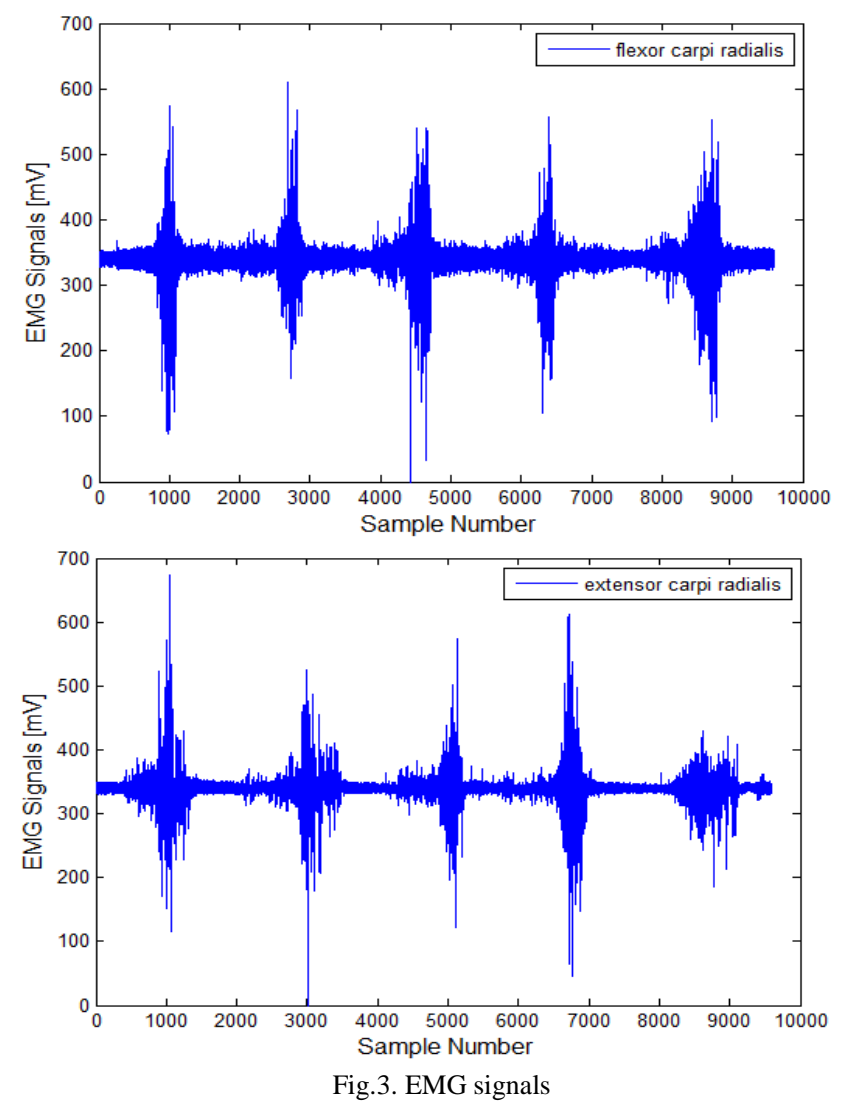

\section{B. Features Extraction}

EMG signals, which are a time series with a random number of elements, are not practical for classification. For this reason, the signal sequences must be matched to the vectors called the feature. In order to successfully classify EMG signals, the feature vectors must be well chosen. There are many feature extraction methods, such as mean absolute value, root mean square, variance etc. The advantages and disadvantages of each method are available.

To classify the EMG signal, it is necessary to extract the features from the signal. Since the recorded signal is measured by different time-dependent movements, it is necessary to analyze it at different intervals of time. For this, the EMG signal is divided into 256 data windows, and different feature extraction of each window is performed by three different methods. In this study, root mean square (RMS), waveform length (WL) and kurtosis (Kurt) methods were used for feature extraction. The analysis using these methods have shown repeatable and discrete model at different states of muscle contraction.

\section{1) Root Mean Square (RMS)}

It is a statistical method used to measure the magnitude of variable quantities. It is particularly useful in waves where the change is positive and negative. A continuously changing function can be calculated for the continuous value series. The root mean square method is calculated as in Equation 1.

$$
R M S=\sqrt{\frac{1}{N} \sum_{n-1}^{N} x_{n}^{2}}
$$


2) Wavelet Length (WL)

Waveform length represents wave cumulative length during time. WL is associated with pulse width, frequency and time. Waveform length method is calculated as shown in Equation 2.

\section{3) Kurtosis (Kurt)}

$$
W L=\sum_{n=1}^{N-1}\left|x_{n+1}-x_{n}\right|
$$

Kurtosis is a measure of data that provides information about the status of the peak. A near-zero kurtosis forms a near normal distribution. A positive value for the kurtosis is a sign of a more normal distribution. Kurtosis method is calculated as shown in Equation 3.

$$
\text { kurtosis }=\frac{\sum_{i=1}^{N}\left(x_{i}-x\right)^{4}}{(N-1) \delta^{4}}
$$

\section{FUZZY LOGIC CLASSIFIER DESIGN}

The fuzzy logic classifier is designed according to the connection between the start and end times of wrist flexion and extension movements. There are a total of 6 entry membership functions, 3 for each muscle in the system. These membership functions are obtained as a result of the feature extractions from the sEMG signals. The signals were analyzed using root mean square as the main method. Other features were applied waveform length and kurtosis. For the classifier design, the state of the contraction information is namely Start (S), Middle (M) and End (E) in determining the final output.

The fuzzy logic classifier has 6 inputs generated from feature extraction of the EMG signals and 1 input which is the states. The block diagram of the fuzzy logic classifier is shown in Figure 4 and Mamdani type fuzzy logic system was used. Triangular shapes are used for the membership function of the inputs and output. The centroid method is used for the defuzzification. The fuzzy logic classifier is developed using Matlab\&Fuzzy Logic Toolbox. The system is tested using Simulink.

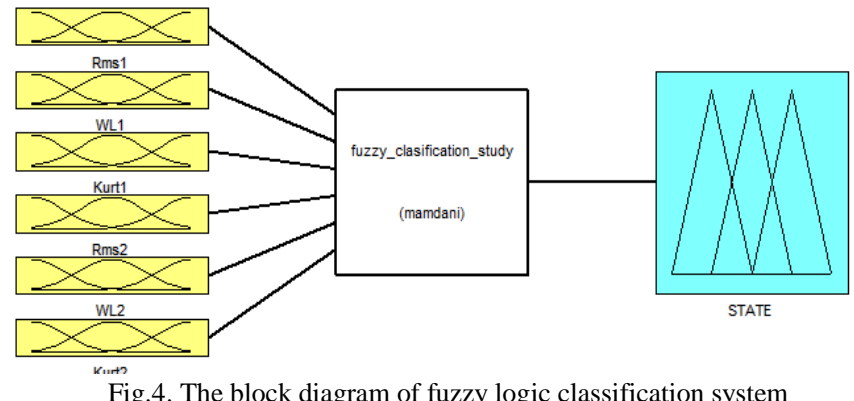

The feature extraction of the sEMG signals gives 6 features. The features that are obtained in case of flexion of the wrist can be describes as Rms1, WL1, Kurt1. The features that are obtained in case of extension of the wrist can be describes as Rms2, WL2, Kurt2. For the input MF, the states of a contraction is used as the fuzzy set which are Start (S), Middle (M), End (E) and Relax (R). The output membership functions are expressed as State1 (S1), State2 (S2) and State3 (S3). Figure 5 shows input and output membership functions.

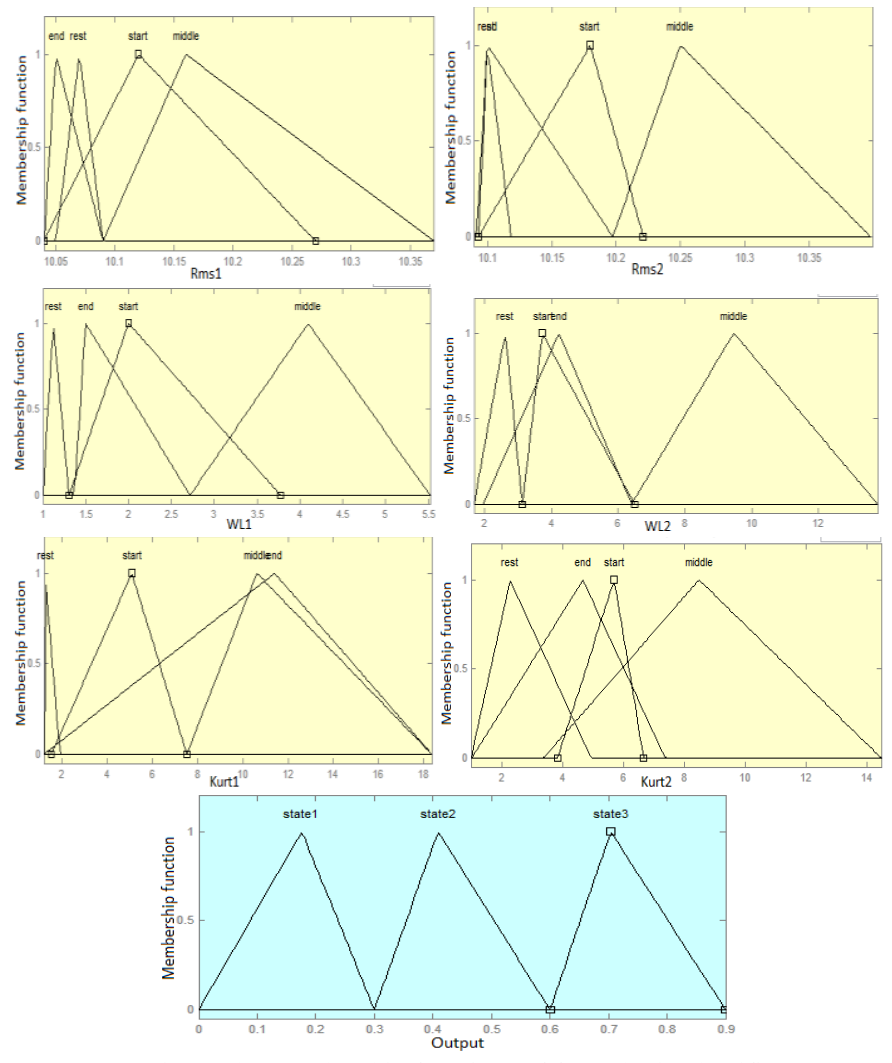

Fig.5. The membership functions of fuzzy logic classifier

Fuzzy logic classifier output states can be described as follows:

1) Wrist flexion of the Start $-\mathrm{S} 3$

2) Wrist flexion of the Middle $-\mathrm{S} 2$

3) Wrist flexion of the End - S1

4) Wrist extension of the Start $-\mathrm{S} 1$

5) Wrist extension of the Middle - S2

6) Wrist extension of the End - S3

The rules are given in Table 1 . The first and second rule is only applied to wrist flexion, rule 5 and 6 apply to wrist extension and rule 3 and 4 apply to wrist flexion/extension. Expert guidance was used while plotting the rule table. The features was expressed Rms1 (A1), WL1 (B1), Kurt1 (C1), Rms2 (A2), WL2 (B2) and Kurt2 (C2) in Table 1.

TABLE I

FUZZY LOGIC CLASSIFIER RULE TABLE

\begin{tabular}{|c|c|c|c|c|c|c|c|c|}
\hline Rule & A1 & B1 & C1 & A2 & B2 & C2 & & State \\
\hline $\mathbf{1}$ & S & S & S & R & R & R & THEN & S3 \\
\hline $\mathbf{2}$ & S & S & S & E & E & E & THEN & S3 \\
\hline $\mathbf{3}$ & M & M & M & R & R & R & THEN & S2 \\
\hline $\mathbf{4}$ & R & R & R & M & M & M & THEN & S2 \\
\hline $\mathbf{5}$ & E & E & E & S & S & S & THEN & S1 \\
\hline $\mathbf{6}$ & R & R & R & S & S & S & THEN & S1 \\
\hline
\end{tabular}

\section{RESULTS AND ANALYSIS}

Feature extraction signals which used as input membership functions in fuzzy logic classification and classification results are shown in Figure 6. EMG signals obtained from flexor carpi radialis muscle were obtained as follows: Rms1, WL1 and Kurt1. EMG signals obtained from extensor carpi radialis muscle were obtained as follows: Rms2, WL2 and Kurt2. 
According to the output graph obtained, the fuzzy logic classifier classifies the EMG signals for three different states (S1, S2, S3).
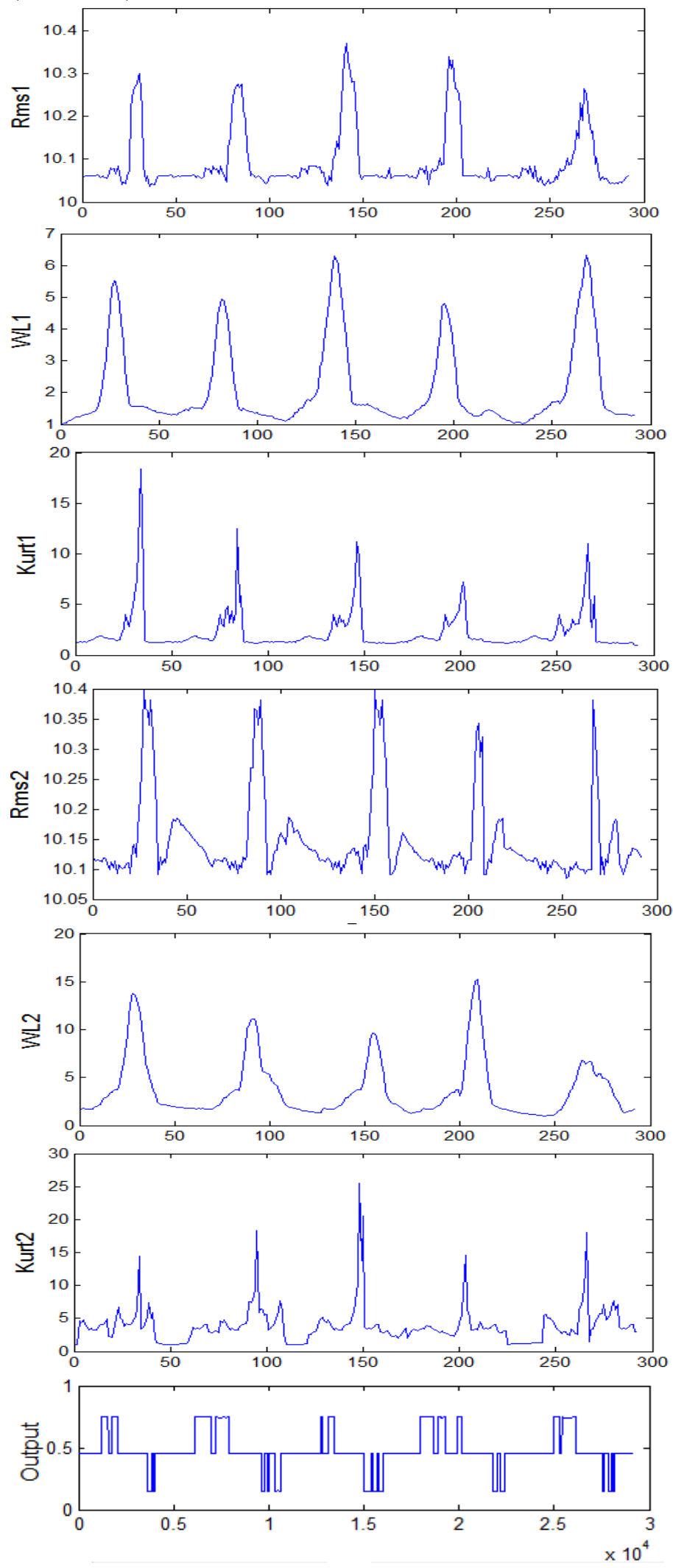

Fig.6. The result of fuzzy logic classifier

The classifier outputs are presented in detail in Figure 7 for initial contraction. The wrist was selected as S3 (0.6-0.9) for the beginning of contraction, S2 (0.3-0.6) for contraction center and
S1 (0-0.3) for the ending of contraction.

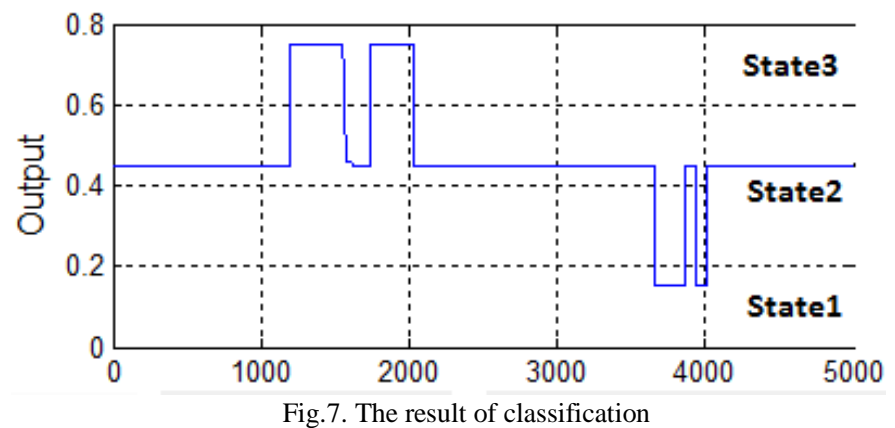

The accuracy (in \%) of the data obtained as a result of the data obtained as a result of the classification are shown in Table 2.

TABLE II

THE ACCURACY (IN \%) OF FUZZY LOGIC CLASSIFIER

\begin{tabular}{cc}
\hline Wrist Flexion/Extension & $\begin{array}{c}\text { \% Classification } \\
\text { success }\end{array}$ \\
\hline Start & 88.84 \\
Middle & 99.85 \\
End & 90.67 \\
Average success & 93.12 \\
\hline
\end{tabular}

\section{CONCLUSION}

In order to understand contraction state movement by using gripping movements, state-based fuzzy logic classification method is used. Conditions are expressed as the beginning, middle and end of the contraction. In this study, the feature extraction and classification of EMG signals received with two channel surface electrodes has been successfully accomplished. As a result of the classification, the wrist flexion / extension times of the user have been determined.

The most important reason of preferring the fuzzy logic classification method is that it does not require any training and its application is simple. A prosthesis developed with the fuzzy logic classification method can easily be transferred from one person to another.

\section{REFERENCES}

[1] M. Diakides, J.D. Bronzino, D.R. Peterson, "Medical Infrared Imaging: Principles and Practices", CRC press, 2012.

[2] E. Criswell, "Cram's introduction to surface electromyography", Jones \& Bartlett Publishers, 2010.

[3] J. He, D. Zhang, N. Jiang, X. Sheng, D. Farina, X. Zhu, "User adaptation in long-term, open-loop myoelectric training: implications for EMG pattern recognition in prosthesis control", Journal of Neural Engineering, vol. 12 , no. $4,2015$.

[4] K. Englehart, B. Hudgins, P.A. Parker, M. Stevenson, "Classification of the myoelectric signal using time-frequency based representations", Medical Engineering \& Physics, vol. 21, no. 6, pp. 431-438, 1999.

[5] X. Chen, D. Zhang, X. Zhu, "Application of a self-enhancing classification method to electromyography pattern recognition for multifunctional prosthesis control", Journal of Neuroengineering and Rehabilitation, vol. 10, no. 1, 2013.Electromagnetic fields in the occupational and general environment, (2011); The $10 \mathrm{kHz}-300 \mathrm{GHz}$ frequency bands normalized parameters values and measurement requirements, HN 80, No.V-199, 2011.

[6] H.J. Fariman, S.A. Ahmad, M.H. Marhaba, M.A.J. Ghasab, P.H. Chappell, "Simple and computationally efficient movement classification approach for EMG-controlled prosthetic hand: ANFIS vs. 
Artificial Neural Network", Intelligent Automation \& Soft Computing, vol. 21, no. 4, pp. 559-573, 2015.

[7] M. Ariyanto, W. Caesarendra, K.A. Mustaqim, M. Irfan, J.A. Pakpahan, J.D. Setiawan, A.R. Winoto, "Finger movement pattern recognition method using artificial neural network based on electromyography (EMG) sensor", in Automation, Cognitive Science, Optics, Micro Electro-Mechanical System, and Information Technology (ICACOMIT), 2015, International Conference, pp. 12-17.

[8] Q. Wu, J.F. Mao, C.F. Wei, S. Fu, R. Law, L. Ding, C.H. Yang, "Hybrid BF-PSO and fuzzy support vector machine for diagnosis of fatigue status using EMG signal features", Neurocomputing, vol. 173, no. 3, pp. 483$500,2016$.

[9] F. Rajablou, M. Ghanbari, "Identifying the condition of arm through classification of EMG signals by the use of hybrid trained adaptive neural fuzzy inference systems (ANFIS)", Bulletin of the Georgian National Academy of Sciences, vol. 9, no. 2, pp. 404-410, 2015.

[10] A. Subasi, "Classification of EMG signals using combined features and soft computing techniques", Applied soft Computing, vol. 12, no. 8, pp. 2188-2198, 2012.

[11] H.B. Xie, H. Huang, J. Wu, L. Liu, “A comparative study of surface EMG classification by fuzzy relevance vector machine and fuzzy support vector machine", Physiological Measurement, vol. 36, no. 2, pp. 191206, 2015.

[12] P. Konrad, "The abc of EMG: A practical introduction to kinesiological electromyography”, Noraxon Inc., 2005.

\section{BIOGRAPHIES}

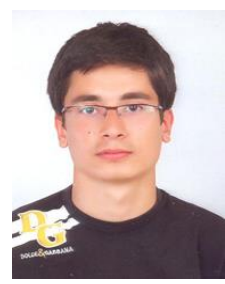

Osman ÜLKİR was born in Hatay, Turkey. He graduated from Kocaeli University, Engineering Faculty, Kocaeli, in 2012, and received M. Sc. degree from Marmara University, Institute of Pure and Applied Sciences, in İstanbul, Turkey, in 2015. He has been started Ph. D. studying in department of Mechatronics Engineering, Marmara University, Institute of Pure and Applied Sciences in 2015 . He is currently working as a research assistant in mechatronics engineering department in technology faculty at Marmara University. His special field of interest Biomechatronics systems, signal processing, lower limb orthosis, and data-driven predictive control.

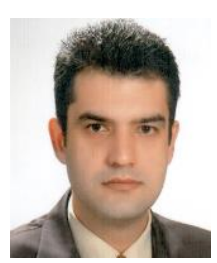

Gökhan GÖKMEN was born in Mersin, Turkey 1974. He received B.S, M.S and PhD degrees from Marmara University, Istanbul, Turkey. He has been working as an associate professor at Marmara University. His current interests are measurement method, signal processing techniques, vocational education.

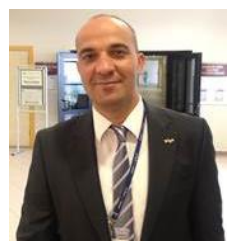

Erkan KAPLANOĞLU received the bachelor's degree from Marmara University Electric Department, in 1996. $\mathrm{He}$ completed his M. Sc. and Ph. D. thesis at Computer Control Department of Marmara University, Institute for Graduate Studies in Pure and Applied Sciences, in 2000 and 2006, respectively. In March 2014, Dr. Kaplanoğlu became Associate Professor at Marmara University, Mechatronics Department. He has been awarded YGGGDRASIL Award (Young Guest Doctoral Researchers in Norway) in 2010. He worked as research assistant professor at Department of Mechanical Engineering Vanderbilt University Faculty of Engineering Nashville/TN, USA from 2011 to 2012. His research areas are Biomechatronics systems, Lower limb Prosthesis, modeling, identification and control. Multivariable, Model Predictive Control, Data Acquisition Systems. 